\title{
Riesgo sanitario de la población vulnerable expuesta al arsénico en la provincia de Buenos Aires, Argentina
}

\author{
Julio A. Navoni, ${ }^{1}$ Diana De Pietri, ${ }^{2}$ Susana Garcia ${ }^{2}$ \\ y Edda C. Villaamil Lepori ${ }^{1}$
}

Forma de citar Navoni JA, De Pietri D, Garcia S, Villaamil Lepori EC. Riesgo sanitario de la población vulnerable expuesta al arsénico en la provincia de Buenos Aires, Argentina. Rev Panam Salud Publica. 2012;31(1):1-8.

RESUMEN Objetivo. Analizar la concentración de arsénico en agua recolectada en localidades de la provincia de Buenos Aires, Argentina, y su relación epidemiológica con factores de susceptibilidad y patologías asociadas.

Métodos. Se cuantificó la concentración de arsénico en 152 muestras provenientes de 52 localidades de Buenos Aires durante el período 2003-2008 mediante generación de hidrurosespectrofotometría de absorción atómica. Se construyó un índice compuesto de salud (ICS) considerando el contenido de arsénico, el porcentaje de hogares con necesidades básicas insatisfechas (NBI) y el de viviendas sin acceso al agua de red. A partir del ICS se definieron zonas de riesgo que fueron asociadas con la mortalidad por tumores malignos relacionados con el arsénico.

Resultados. Las concentraciones de arsénico se ubicaron en un rango amplio, desde 0,3 hasta $187 \mu \mathrm{g} / \mathrm{L}$, con una mediana de $40 \mu \mathrm{g} / \mathrm{L}$. El 82\% de las muestras presentaron niveles de arsénico superiores al valor límite aceptable de $10 \mu \mathrm{g} / \mathrm{L}$, y más de la mitad de ellas provenían de agua de red. La mortalidad promedio (defunciones/100000 habitantes) por tumores en los departamentos estudiados fue mayor en los varones que en las mujeres: vías respiratorias (310 frente a 76), vias urinarias (44 frente a 11) y piel (21 frente a 11), respectivamente. Las regiones de mayor concentración de arsénico y pobreza, junto con la falta de agua de red, presentaron un riesgo relativo incrementado de 2 a 4 veces.

Conclusiones. La caracterización realizada a través del índice compuesto de salud sintetizó el riesgo sanitario de la exposición al arsénico de la población con niveles de carencia socioeconómica de una amplia región de la provincia de Buenos Aires.

Palabras clave Arsénico; intoxicación por arsénico; medición de riesgo; control de la contaminación del agua; áreas de pobreza; Argentina.

1 Universidad de Buenos Aires, Facultad de Farmacia y Bioquímica, Cátedra de Toxicología y Química Legal, Buenos Aires, Argentina.

2 Ministerio de Salud de la Nación, Dirección Nacional de Determinantes de la Salud e Investigación, Buenos Aires, Argentina. La correspondencia se debe enviar a Diana De Pietri, depietrid@hotmail.com
La presencia de arsénico en el agua subterránea de grandes extensiones territoriales constituye un problema de salud pública debido a su toxicidad. El peligro proviene de la extracción del agua mediante pozos localizados en zonas geológicas ricas en arsénico y su uso tanto en actividades domésticas como agropecuarias. La ingesta de arsénico en el agua de bebida durante largos períodos de tiempo se ha asociado con una enfermedad denominada hidroarsenicismo crónico regional endémico (HACRE), que se caracteriza por lesiones en la piel 
y alteraciones sistémicas cancerosas y no cancerosas (1). Entre las alteraciones en la salud se incluyen hiperhidrosis e hiperqueratosis palmoplantar, pérdida de la pigmentación cutánea, trastornos vasculares y metabólicos, y cáncer de piel, vejiga y pulmón, entre otros $(2,3)$.

Los efectos adversos del arsénico para la salud dependen de la dosis y la duración de la exposición (4). Aunque no existe una concentración de arsénico que se considere segura, se ha establecido un nivel guía para la calidad del agua de bebida de 10 partes por billón (ppb) en el agua de consumo diario (bebida y preparación de alimentos) (5). Las acciones de salud están dirigidas a evitar o discontinuar la exposición. En las etapas preclínicas y clínicas del HACRE la recuperación puede ser completa si se reemplaza el agua arsenical por agua segura. En la última etapa las manifestaciones no cancerosas pueden ser reversibles, pero cuando aparecen neoplasias malignas, la única medida es el tratamiento oportuno para reducir las secuelas (1).

La sensibilidad humana a los efectos tóxicos del arsénico varía, probablemente debido a factores genéticos, metabólicos, de la dieta, del estado de salud, del sexo y de la edad, entre otros. La situación de pobreza puede incrementar la susceptibilidad de la población a enfermar por exposiciones a uno o más tóxicos ambientales (3, 4, 6, 7). Asimismo, el menor acceso a la atención médica oportuna hace que el estado de pobreza sea un factor de vulnerabilidad, lo que incrementa el riesgo de mortalidad.

Se estima que en América Latina la población expuesta al arsénico es de 4,8 millones de personas, de la cual cerca de la mitad se encuentra en la Argentina $(5,8-10)$. La mayoría de los casos reportados de HACRE en la Argentina provienen de poblaciones rurales dispersas expuestas a elevados contenidos de arsénico, que consumen agua sin tratamiento o control de calidad (8). La información de mortalidad por cáncer y su vinculación con la exposición ambiental al arsénico es escasa, especialmente en lo que respecta a concentraciones de arsénico en el agua de bebida cercanas o inferiores a $100 \mu \mathrm{g} / \mathrm{L}$.

El presente trabajo estuvo dirigido a determinar el nivel de arsénico en el agua de bebida en localidades de la provincia de Buenos Aires, construir un índice compuesto de salud (ICS) para medir el riesgo sanitario cuando se combina la exposición a un tóxico y la vulnerabilidad social, y evaluar las tasas de mortalidad por tumores relacionados con la exposición al arsénico en esas localidades.

\section{MATERIALES Y MÉTODOS}

El estudio realizado es de tipo ecológico, y la unidad de análisis corresponde a grupos poblaciones localizados en diferentes departamentos de Buenos Aires.

Para seleccionar las 52 localidades se consideraron las muestras de agua remitidas al laboratorio del Centro de Asesoramiento Toxicológico Analítico (CENATOXA) para la determinación del contenido de arsénico durante el período 2003-2008.

Las localidades de la provincia de Buenos Aires pertenecen hidrogeológicamente a la llanura Chacopampeana (11).

\section{Determinación del arsénico total en el agua}

Se recolectaron como muestra alícuotas de $250 \mathrm{~mL}$ de agua en envases plásticos limpios siguiendo las indicaciones del laboratorio (12). Una vez remitidas al laboratorio, las muestras se acidificaron con $\mathrm{HNO}_{3}$ y se conservaron a $4{ }^{\circ} \mathrm{C}$ hasta su análisis, que fue realizado dentro de la semana de su recepción.

La determinación de arsénico en el agua fue realizada con un espectrofotómetro de absorción atómica, modelo AA 475, Varian, equipado con un sistema de generación de hidruros VGA77, Varian. La atomización térmica fue realizada en una celda de cuarzo calentada mediante una llama de aire/acetileno.

Para la preparación de reactivos y estándares se utilizó agua desionizada (18,2 M $\Omega . c m)$. Todos los reactivos fueron de calidad analítica. Las soluciones estándares de trabajo de $\left[\mathrm{As}^{\mathrm{V}}\right]$ fueron preparadas diariamente por diluciones sucesivas de una solución stock de $1000 \mu \mathrm{g} / \mathrm{L}$ Titrisol (Merck). Los reactivos químicos incluyeron ácido clorhídrico (Merck), ácido ascórbico (Merck), yoduro de potasio (Merck), tetrahidroborato de sodio (Merck) e hidróxido de sodio (Merck). El nitrógeno, el aire y el acetileno fueron de calidad ultrapura (Indura). Todo el material de vidrio y plástico utilizado fue tratado con solución de detergente no iónico alcalino durante 12 h y $\mathrm{HNO}_{3}$ al 10\% (v/v) durante
12 h, y después fue enjuagado tres veces con agua desionizada y secado al aire.

La determinación de arsénico total fue realizada a partir del método descrito por Muñoz et al. (13). Las muestras fueron filtradas a través de una membrana de $0,45 \mu \mathrm{m}$. A una alícuota de estas $(2,5 \mathrm{~mL})$ se les adicionó igual volumen de $\mathrm{HCl}$ concentrado. Las muestras estándares fueron prerreducidas mediante el agregado de $5 \mathrm{~mL}$ de una solución al $5 \% \mathrm{p} / \mathrm{v}$ de yoduro de potasio y $5 \%$ $\mathrm{p} / \mathrm{v}$ de ácido ascórbico, mantenidas a temperatura ambiente por 30 minutos y luego llevadas a un volumen final de $25 \mathrm{~mL}$ en un matraz aforado con $\mathrm{HCl}$ 6M. La cuantificación se realizó mediante interpolación en curva de calibración externa. Las condiciones optimizadas de generación de arsinas fueron $\mathrm{NaBH}_{4}$ al $0,7 \%(\mathrm{p} / \mathrm{v})$ en $\mathrm{NaOH}$ al $0,5 \%(\mathrm{p} / \mathrm{v})$

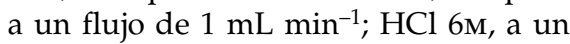
flujo de $1 \mathrm{~mL} \mathrm{~min}^{-1}$, y muestras/estándares a $5 \mathrm{~mL} \mathrm{~min}^{-1}$ utilizando nitrógeno como gas transportador. Las condiciones de lectura fueron longitud de onda, 193,7 nm; apertura de rendija, 0,5 nm; fuente de energía, lámpara de cátodo hueco con una intensidad de corriente de $6 \mathrm{~mA}$; corrección de fondo con lámpara de deuterio.

En cada secuencia de cinco muestras analizadas por duplicado se incluyeron blancos de reactivos y materiales de referencia. Las características analíticas del método fueron límite de detección 0,3 $\mu \mathrm{g} / \mathrm{L}$, límite de cuantificación 0,6 $\mu \mathrm{g} / \mathrm{L}$ y precisión intermedia 5\%. La exactitud de la medición se evaluó mediante materiales certificados para elementos traza en agua EP-H-2 EnviroMAT. No hubo diferencias significativas entre el valor certificado y el obtenido (sesgo promedio 1,3\%).

\section{Medición del riesgo ambiental}

Se georreferenció la condición sociodemográfica de los departamentos de la provincia de Buenos Aires a través del porcentaje de hogares con necesidades básicas insatisfechas (NBI) (14) y el de viviendas sin provisión de agua de red. La base cartográfica digital de la provincia y los datos de población y vivienda provienen del censo nacional 2001 (15) Los valores de arsénico en agua fueron georreferenciados a nivel de las localidades de Buenos Aires e integrados con la información precedente. 
La integración de las variables consideradas como indicadoras de riesgo sanitario de exposición al arsénico (hogares con NBI, viviendas sin agua de red y arsénico en agua de bebida) fue realizada mediante el índice compuesto de salud (ICS), provisto en el programa SIG-Epi (OPS/OMS, Estados Unidos) (16).

El ICS está basado en puntajes $Z$, que se calculan a partir de estimar el valor medio y el desvío estándar del rango de valores de cada indicador según la relación $\mathrm{Z}=(\mathrm{I}-\mathrm{m}) / \mathrm{T}$, donde $I$ es el valor del indicador, $m$ es la media y $T$ el desvío estándar. El índice es la suma de todos los $Z$ de cada indicador según ICS = $(\mathrm{aZ1}+/-\mathrm{bZ2}+/-\mathrm{cZ3})$, donde la letra minúscula es la influencia o peso (ponderación) del indicador expresado en porcentaje, y el signo $+\mathrm{o}-\mathrm{el}$ aporte del indicador a dicho escenario. El aporte de cada indicador a la definición del riesgo fue realizado en forma diferencial. A tal efecto se asignó la mayor influencia al contenido de arsénico en el agua de bebida y a la condición de pobreza, y en menor medida a la falta de provisión de agua por red.

Se realizó una representación gráfica de los valores de ICS con el objeto de estratificar el territorio en zonas con diferente nivel de riesgo ambiental para poder establecer comparaciones, y así obtener una estimación de la diferencia, no sesgada, entre los niveles de riesgo. Los departamentos se clasificaron como "sin exposición" o "expuestos", según el nivel de riesgo sanitario.

La clasificación de la población en "sana" o "enferma" para completar la tabla de contingencia de "exposiciónenfermedad" se extrajo de la base de datos de mortalidad de la Dirección de Estadística e Información de Salud del Ministerio de Salud de la Nación. La base de datos se encuentra categorizada según la clasificación internacional de enfermedades (CIE-10) (17).

Se calcularon las tasas de mortalidad a partir del cociente entre el número promedio de fallecidos mayores de 15 años según el sexo durante el período 2001-2006 y el número de habitantes de esos grupos poblacionales de cada uno de los departamentos. Las tasas se expresaron por cada 100000 habitantes.

El análisis estadístico se llevó a cabo con un nivel de significación de $\alpha=0,05$. Se emplearon los programas SPSS 10.0 (versión estándar, IBM, Estados Unidos),
SIGEpi 3.2 y EPIDAT 3.0 (Xunta de Galicia; OPS, Estados Unidos) (18).

\section{RESULTADOS}

Se encontró un amplio rango de concentraciones de arsénico en el agua de bebida de las 152 muestras estudiadas con un valor promedio de 49,9 $\mu \mathrm{g} / \mathrm{L}, \mathrm{y}$ en algunas localidades la concentración alcanzó valores cercanos a los $200 \mu \mathrm{g} / \mathrm{L}$ (figura 1). Del total de muestras, cerca de $40 \%$ superaron el valor máximo de $50 \mu \mathrm{g} / \mathrm{L}$ y $80 \%$ el de $10 \mu \mathrm{g} / \mathrm{L}$. Según la forma de suministro, las muestras analizadas se agruparon en "red", "perforación" y "otras" (sistemas de acopio de agua, como cisternas y aljibes). No se observaron diferencias en función del tipo de provisión de agua (cuadro 1). Tanto las muestras de agua de red como las obtenidas de perforación presentaron niveles de arsénico similares, lo que in- dica una ausencia de pretratamiento, al menos para este elemento.

Los niveles de arsénico más elevados se distribuyeron en departamentos con preponderancia de población rural; sin embargo, se detectaron valores intermedios en departamentos con alto número de habitantes urbanos (por ej., Junín, 9 de julio, Chacabuco) (figura 2).

La estratificación del territorio se realizó según criterios de peligro (exposición al arsénico) y vulnerabilidad (condición de pobreza), ya que no se observó relación o jerarquía entre variables (coeficientes de correlación de Pearson [95\%]: arsénico en agua de bebida frente a hogares con NBI [-0,31]; arsénico en agua de bebida frente a viviendas sin agua de red $[-0,02]$ y hogares con NBI frente a viviendas sin agua de red $[0,22]$ ).

El ICS permitió caracterizar y representar los departamentos estudiados según tres niveles de riesgo: bajo, medio

FIGURA 1. Distribución del contenido de arsénico en el agua de bebida de 152 muestras provenientes de localidades de la provincia de Buenos Aires, Argentina, 2010

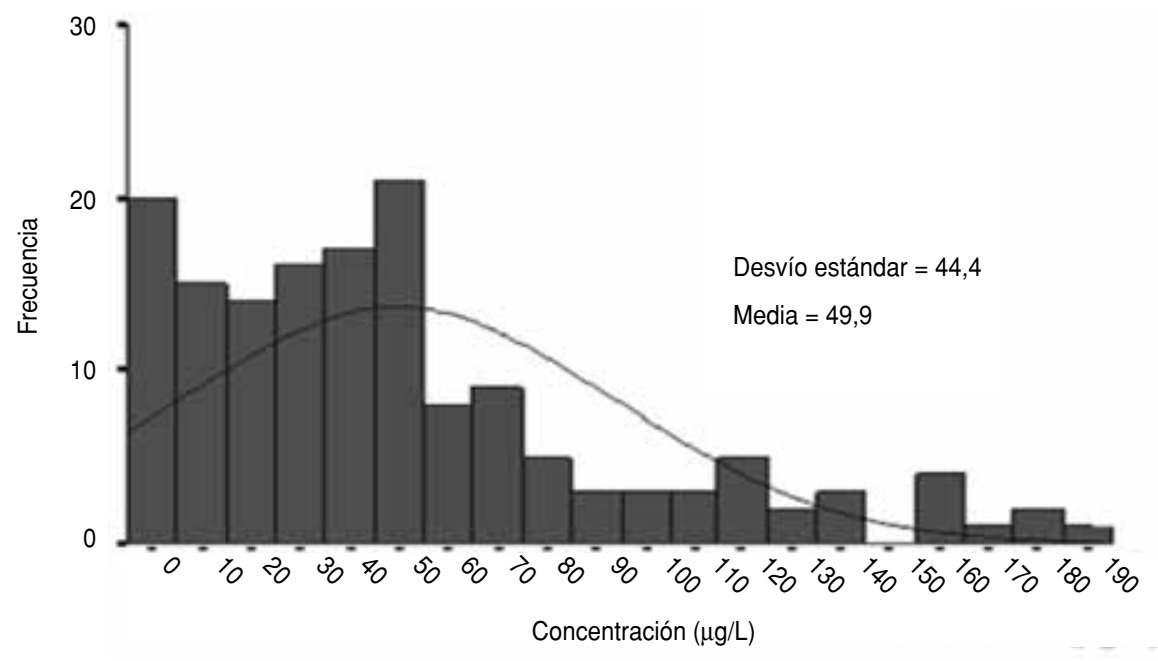

CUADRO 1. Contenido de arsénico en el agua de bebida en función del tipo de provisión de agua, Buenos Aires, Argentina, 2010

\begin{tabular}{lccccc}
\hline & Total & Perforación & Red & Otras & s/d \\
\hline Número de muestras & 152 & 53 & 75 & 8 & 16 \\
Promedio $(\mu \mathrm{g} / \mathrm{L})$ & 49,9 & 45,8 & 51,8 & 47,9 & 54,0 \\
Mediana $(\mu \mathrm{g} / \mathrm{L})$ & 40,1 & 33,6 & 44,4 & 68,0 & 52,0 \\
Mínimo $(\mu \mathrm{g} / \mathrm{L})$ & $\mathrm{ND}$ & $\mathrm{ND}$ & $\mathrm{ND}$ & $\mathrm{ND}$ & 5,0 \\
Máximo $(\mu \mathrm{g} / \mathrm{L})$ & 187,2 & 187,2 & 183,2 & 70,0 & 139,0 \\
Porcentaje & 100 & 35 & 49 & 5 & 11
\end{tabular}

Perforación: extracción de agua subterránea a través de pozos individuales; Red: viviendas con servicio de agua de red; Otras: Obtención de agua por sistemas de acopio como aljibes y cisternas; s/d: sin datos del tipo de suministro; ND: no disponible. 
FIGURA 2. Caracterización sociodemográfica de los departamentos de la provincia de Buenos Aires y de las localidades estudiadas en relación al contenido de arsénico en el agua de bebida, Buenos Aires, Argentina, 2010
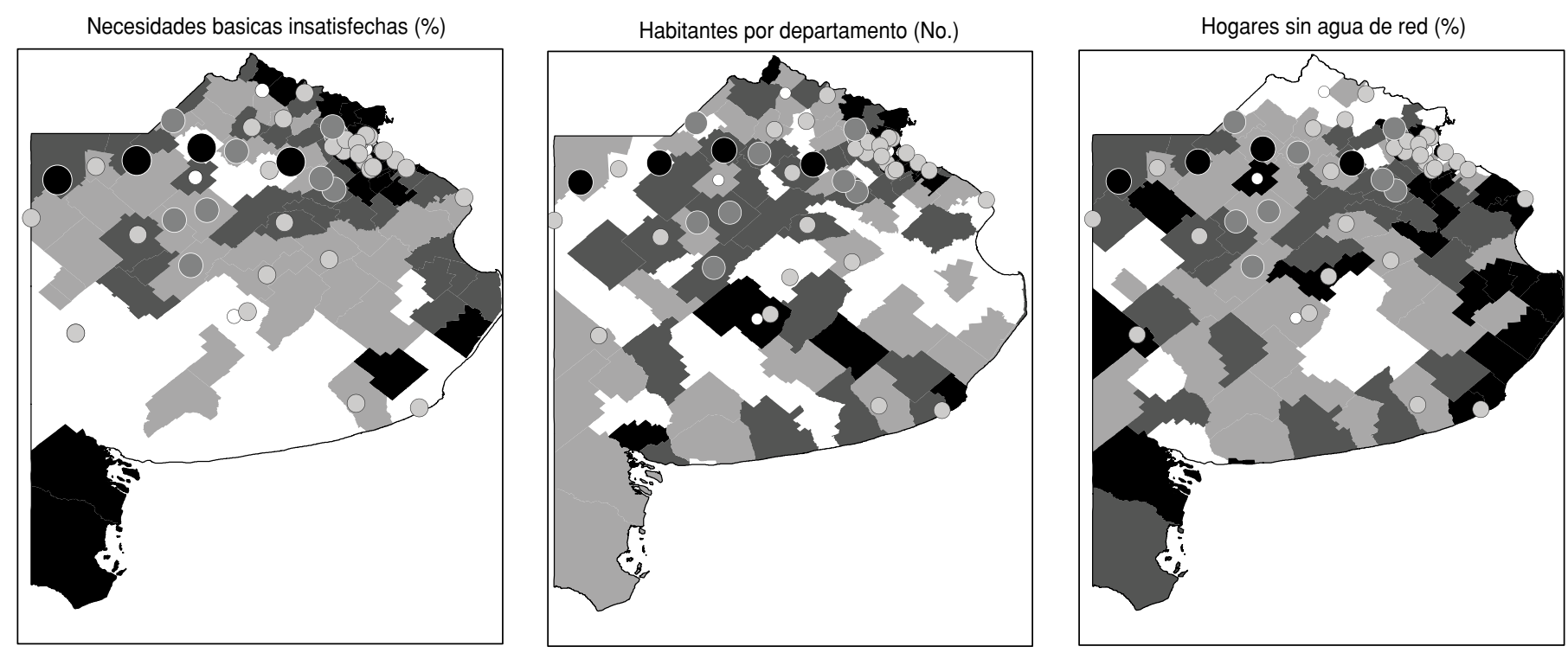

Arsénico en agua de bebida $(\mu \mathrm{g} / \mathrm{L})$

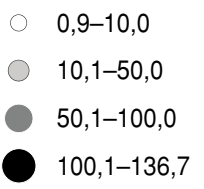

Indicador por departamentos de Buenos Aires

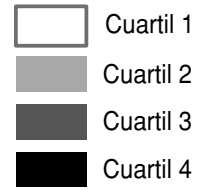

Fuente: Censo Nacional de Población, Hogares y Viviendas 2001.

y alto (figura 3, A). La zona de mayor riesgo quedó definida por los sitios que presentaron una elevada concentración de arsénico en el agua de bebida, además de una alta proporción de hogares pobres.

La concentración promedio de arsénico en el agua de bebida en la zona de bajo riesgo fue menor de $17 \mu \mathrm{g} / \mathrm{L}$, en tanto que las muestras de los departamentos caracterizados como de riesgo medio y alto presentaron valores superiores a $43 \mu \mathrm{g} / \mathrm{L}$. Si bien los valores promedio porcentuales de hogares con NBI hallados para cada categoría de riesgo no difirieron sustancialmente, se observó una mayor proporción de hogares con NBI en la categoría de alto riesgo. La proporción de viviendas sin agua de red se incrementó levemente con el nivel de riesgo (figura $3, \mathrm{~B}$ y $\mathrm{C}$ ).

La categoría de riesgo bajo incluye a la Ciudad de Buenos Aires, y su elevada población sesga las categorías en función del número de habitantes. Por este motivo, se definió como zona de exposición a los departamentos incluidos en las categorías de riesgo medio y alto.
La población total de la región considerada en este estudio fue de 7307977 habitantes (mayores de 15 años de edad), de los cuales 2127410 correspondieron a mujeres y 1819843 a varones de zonas no expuestas, frente a 1719263 y 1641461 de zonas de exposición, respectivamente. En la figura 4 se describe la información epidemiológica de mortalidad por cáncer de vías urinarias, piel y vías respiratorias de los 47 departamentos incluidos en este estudio. Los varones presentaron, en relación con las mujeres, una mayor mortalidad en cada una de las patologías analizadas. Las tasas promedio de mortalidad por tumores de vías respiratorias fueron las de mayor diferencia entre sexos (76 mujeres y 310 varones fallecidos por cada 100000 habitantes). La diferencia entre sexos fue menor para las tasas promedio de mortalidad por tumores de vías urinarias y mínima para tumores de piel (figura 4).

Las poblaciones que residen en zonas de riesgo presentaron entre 3 y 4 veces más mortalidad para cada tipo de tumor asociado al arsénico, con respecto a la población que reside en zonas sin riesgo sanitario (cuadro 2).

\section{DISCUSIÓN}

Más del $80 \%$ de las muestras analizadas provenientes de localidades de la provincia de Buenos Aires presentaron concentraciones de arsénico superiores a los valores guía vigentes, con valores máximos equivalentes a 3 veces el valor de $50 \mu \mathrm{g} / \mathrm{L}$ y 18 veces el recomendado por la OMS, incorporado a la normativa nacional para su implementación en el año $2012(5,19)$. Los niveles establecidos fueron comparables a los reportados en otras provincias argentinas, como Catamarca (10 a $170 \mu \mathrm{g} / \mathrm{L})(20)$, Chaco (10 a $213 \mu \mathrm{g} / \mathrm{L})$ (21), Córdoba (10 a 450 $\mu \mathrm{g} / \mathrm{L})(22,23)$, Salta $(200 \mu \mathrm{g} / \mathrm{L})(24,25)$, Tucumán, $163 \mu \mathrm{g} / \mathrm{L}$ (26) y Santa Fe (50 a $200 \mu \mathrm{g} / \mathrm{L})(27)$, e inferiores a los encontrados en provincias como La Pampa (4 a $5300 \mu \mathrm{g} / \mathrm{L}$ ) (28) y Santiago del Estero $(10$ a $620 \mu \mathrm{g} / \mathrm{L})(10,29)$.

Uno de los factores analizados fue la provisión de agua a través del sistema de distribución de red. La mayor parte 
FIGURA 3. A. Distribución espacial de las categorías de riesgo sanitario de exposición al arsénico de poblaciones vulnerables según departamentos de la provincia de Buenos Aires. B. Cuadro de los valores promedio. C. Diagrama de cajas de los factores utilizados, Buenos Aires, Argentina, 2010

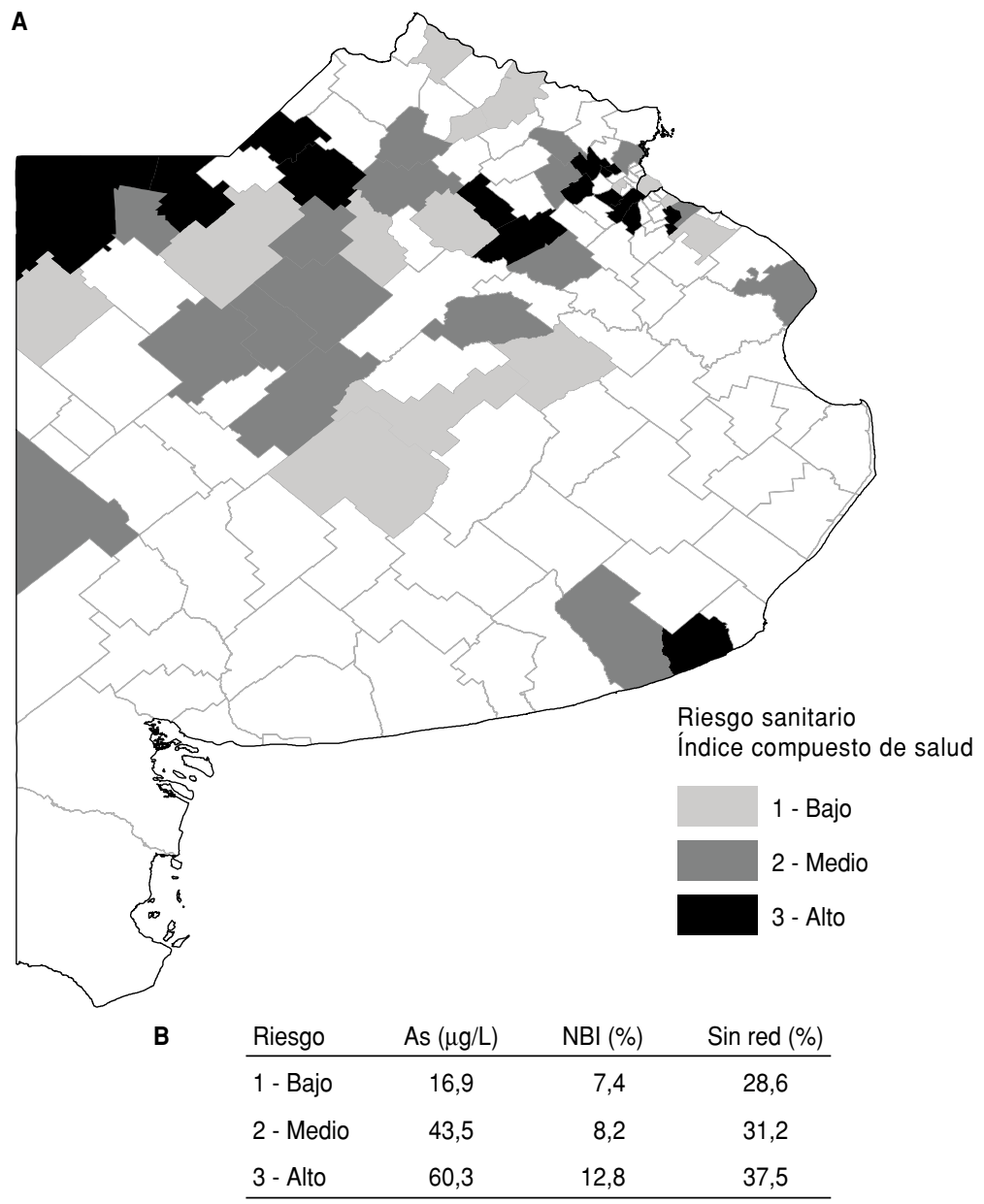

C
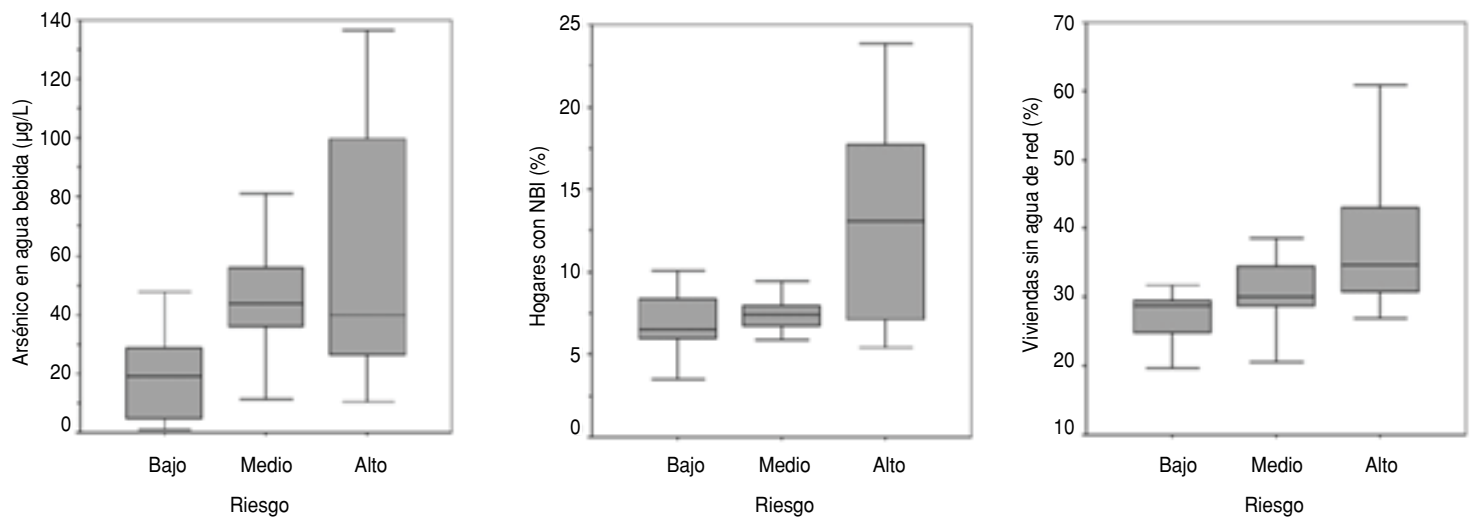

NBI: Necesidades básicas insatisfechas.

de los departamentos incluidos en este estudio obtienen el agua para consumo humano de capas subterráneas. El contenido de arsénico elevado encontrado en dichas muestras indica la ausencia de control de la potabilidad para este elemento. Por consiguiente, las poblaciones, independientemente del tipo de provisión de agua, presentan un nivel de exposición similar.

En este estudio se caracterizó la información de centros urbanos en regiones potencialmente afectadas por el HACRE. Es decir, la población potencialmente afectada pertenece no solo a asentamientos rurales dispersos, como ya es conocido en la bibliografía (4), sino también a centros urbanos. 
FIGURA 4. Diagrama de cajas de las tasas de defunción por 100000 habitantes según el sexo y el tipo de tumores, Buenos Aires, Argentina, 2010

Piel

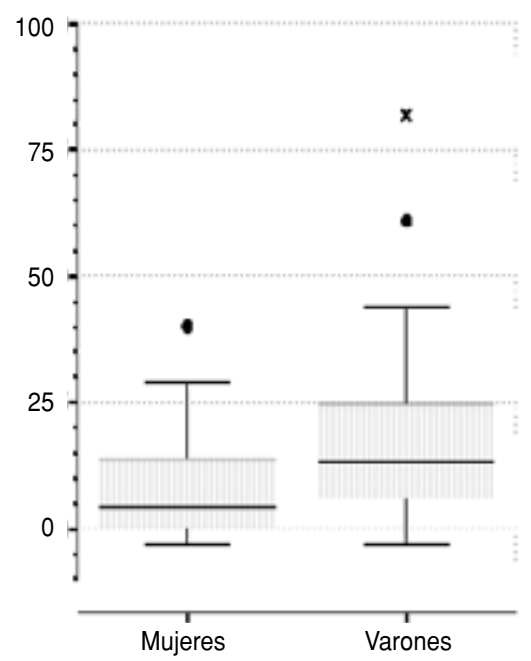

Vías respiratorias

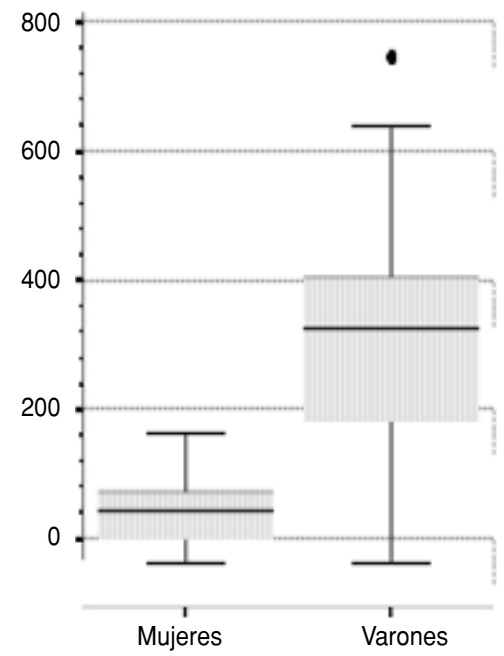

Vías urinarias

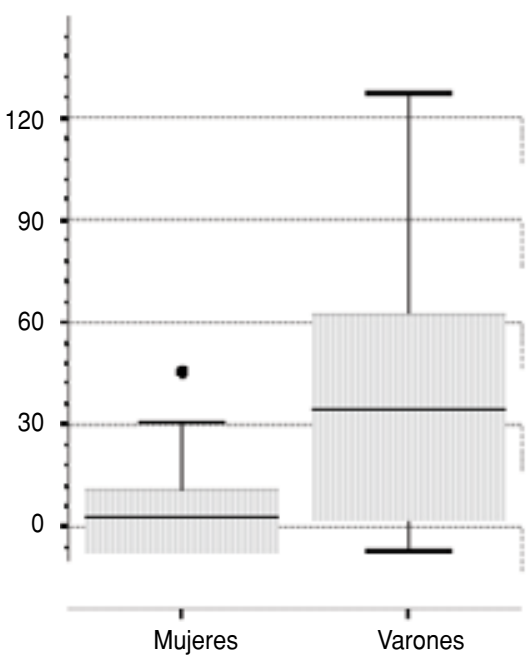

Fuente: Dirección de Estadística e Información de Salud del Ministerio de Salud de la Nación. Nota: los círculos y cruces indican los valores atíícos y extremos, respectivamente.

CUADRO 2. Riesgo relativo de cáncer de piel, de las vías respiratorias y las vías urinarias, Buenos Aires, Argentina, 2010

\begin{tabular}{llcc}
\hline $\begin{array}{c}\text { Mortalidad } \\
\text { por tumores }\end{array}$ & & Riesgo relativo & IC 95\% \\
\hline Piel & Mujeres & 3,9 & $2,9-5,2$ \\
\multirow{2}{*}{ Vías respiratorias } & Varones & 3,1 & $2,5-3,9$ \\
& Mujeres & 3,2 & $2,9-3,5$ \\
Vías urinarias & Varones & 3,0 & $2,8-3,1$ \\
& Mujeres & 4,0 & $3,0-5,2$ \\
& Varones & 3,8 & $3,3-4,4$ \\
\hline
\end{tabular}

IC 95\%: intervalo confianza de $95 \%$.

La utilización del ICS contribuyó a delimitar zonas con diferentes niveles de riesgo ambiental. La estratificación del territorio de Buenos Aires representó zonas de exposición suficientemente discrepantes como para establecer diferencias atribuibles a la exposición al arsénico en poblaciones con bajo nivel socioeconómico con base en la información existente.

Se han descrito asociaciones entre la condición de pobreza y la mortalidad por tumores (30), entre los que figura el cáncer de vejiga y de pulmón, patologías relacionadas también con la toxicidad del arsénico.

En términos de una evaluación de riesgo, la función "exposición/respuesta" fue definida mediante la comparación de poblaciones "expuestas" y "no expuestas", estableciendo niveles de asociación entre la exposición al arsénico en pobla- ciones vulnerables y la mortalidad por cáncer.

Se halló una mayor tasa de mortalidad por cáncer de piel, vías urinarias y vías respiratorias asociada a la exposición arsenical en la población masculina. El cáncer de vías respiratorias presentó los mayores valores de mortalidad y diferencia entre sexos.

Es inequívoca la existencia de una relación dosis-respuesta entre la exposición al arsénico a través del agua de bebida y la aparición de cáncer de piel, vejiga y pulmón $(31,32)$. Sin embargo, esta asociación ha sido demostrada a niveles elevados de exposición (33). Son escasos los reportes de asociación entre prevalencia de cáncer a niveles de exposición menores de $200 \mu \mathrm{g} / \mathrm{L}$. Algunos informes describen un mayor riesgo de cáncer de vejiga (34-36), en tanto que otros indican un incremento en el riesgo de cáncer de pulmón a concentraciones bajas de arsénico en el agua, cercanas a $50 \mu \mathrm{g} / \mathrm{L}$ (34). La mayoría de dichos estudios señalan una acción sinérgica entre el arsénico y el hábito de fumar, y esta variable incide fundamentalmente a niveles bajos de exposición $(35,36)$. El consumo de tabaco en la región estudiada es elevado. Más del $60 \%$ de la población mayor de 18 años fuma o ha fumado a lo largo de su vida. Esta observación indica que dicho hábito podría ser un factor determinante en las observaciones descritas previamente (37).

La piel es el órgano más sensible a la intoxicación crónica por arsénico, y las manifestaciones cutáneas constituyen los primeros hallazgos clínicos del HACRE (6). Recientemente se ha comunicado una contundente relación dosis-respuesta de aparición de manifestaciones cutáneas (hiperqueratosis) a niveles de exposición comparables con los hallados en este trabajo (34). La aparición de neoplasias malignas cutáneas relacionadas con el arsénico ha sido considerada un signo de alerta temprano de un posible y subsecuente desarrollo de cáncer de órganos internos, además de ser los tumores citados con más frecuencia en la bibliografía $(6,38)$. El sexo es una variable asociada con el metabolismo del arsénico, y estaría relacionado con la carcinogenicidad inducida por este elemento (39). Las mujeres presentan un 
perfil de metilación más eficiente (mayor eliminan más fácilmente la carga arsenical incorporada (40). En la bibliografía se describe una menor tasa de casos de cáncer asociado con el arsénico en mujeres (41-44). Los resultados obtenidos en este trabajo coinciden con dichas observaciones, ya que la tasa de mortalidad por cáncer fue mayor en el sexo masculino.

Sin embargo, el riesgo relativo asociado al sexo indicó una mayor susceptibilidad en las mujeres que en los varones en función de la zona donde se habita. Este hecho podría estar asociado a una mayor exposición, determinada en parte por diferencias en el estilo de vida con respecto a los varones. proporción de especies dimetiladas), y

La estratificación a través de un índice que integra las condiciones de pobreza con la exposición al arsénico explica las diferencias observadas con otros trabajos realizados a niveles de exposición comparables $(6,35,39)$.

Una limitación de este trabajo fue no disponer de datos de morbilidad de HACRE para constatar las observaciones alcanzadas mediante la utilización del ICS. De esta manera se podrían identificar los potenciales factores de confusión.

Los resultados obtenidos constituyen criterios guía para la planificación de acciones de prevención y control para la utilización de los recursos de manera racional y eficiente.

\section{REFERENCIAS}

1. Ministerio de Salud de la Nación, Argentina. Programa nacional de prevención y control de las intoxicaciones. Hidroarsenicismo crónico regional endémico. Módulo de capacitación; 2001;3:1-68.

2. Mukherjee A, Sengupta MK, Hossain MA, Ahamed S, Das B, Nayak B, et al. Arsenic contamination in groundwater: A global perspectives with emphasis on the Asian scenario. J Health Popul Nut. 2006;24:143-63.

3. Ng J, Wang J, Shraim A. A global health problem caused by arsenic from natural sources. Chemosphere. 2003;52:1353-9.

4. Agency for toxic substances and disease registry (ATSDR). Toxicological profile for arsenic. Atlanta, GA. US 2007. Disponible en http://www.atsdr.cdc.gov/toxprofiles/TP. asp?id=22\&tid=3

5. World Health Organization. Guidelines for Drinking-Water Quality, Vol. 1. Recommendations. $3^{\mathrm{a}}$ ed; Geneva: WHO; 2004.

6. Kazi TG, Arain MB, Baig MK, Afridi HI, Jalbani N, Sarfraz RA, et al. The correlation of arsenic levels in drinking water with the biological samples of skin disorders. Sci Total Environ. 2009;407:1019-26.

7. Cantor KP, Lubin JH. Arsenic, internal cancers and issues in inference from studies of low-level exposures in human populations. Toxicol Appl Pharmacol. 2007;222:252-7.

8. Bundschuh J, Farias B, Martin R, Storniolo A, Bhattacharya P, Cortes J, et al. Groundwater arsenic in the Chaco-Pampean Plain, Argentina: case study from Robles county, Santiago del Estero Province. Appl Geochem. 2004;19:231-43.

9. Ministerio de Salud de la Nación, Argentina. Resolución 153/2001. Programa de minimización de riesgos por exposición a arsénico en agua de consumo del Departamento de Salud Ambiental de la Dirección de Promoción y Protección de la Salud. Boletín Oficial 2001;29599:19.

10. Villaamil Lepori E, Garcia SI (Ministerio de Salud de la Nación, Argentina). Epidemiología del Hidroarsenicismo Crónico Regio- nal Endémico-Estudio Colaborativo Multicéntrico; 2007. Disponible en http://www. ambiente.gov.ar/archivos/web/UniDA/ File/libro_hidroarsenicismo_completo.pdf Acceso el 20 de agosto de 2010.

11. Auge M. Regiones hidrogeológicas. República Argentina, 2004. Disponible en www.gl.fcen. uba.ar/investigacion/grupos/hidrogeologia/ auge/Reg-Hidrogeo.pdf. Acceso el 13 de febrero de 2011.

12. Clesceri L, Greeberg A y Eaton A (eds.). Standard Methods for the examination of Water and Wastewater. Summary of Special Sampling and Handling Requirements. 20 ed.; [Washinton, Estados Unidos]: Editorial APHA,AWWA,WEF; 1998. Pp. 1-33.

13. Muñoz $O$, Díaz OP, Leyton $I$, Núñez $N$, Devesa V, Súñer MA, et al. Vegetables collected in the cultivated Andean area of the Northern Chile. Total and inorganic arsenic contents in raw vegetables. J Agric Food Chem. 2002;50:642-7.

14. Instituto Nacional de Estadística y Censo, Argentina. Acerca del método utilizado para la medición de la pobreza en Argentina. Disponible en http://www.indec.mecon. $\mathrm{ar} /$ nuevaweb/cuadros/7/sesd-metodologia-pobreza.pdf Acceso el 17 de agosto de 2010.

15. Instituto Nacional de Estadística y Censo, Argentina. Censo Nacional de Población, Hogares y Viviendas 2001. Disponible en http:// www.indec.mecon.ar/webcenso/index.asp Acceso el 17 de agosto de 2010.

16. Organización Panamericana de la Salud. Sistema de información geográfica en epidemiología y salud pública. Manual del usuario. Versión 1.26. Washington, D.C.: OPS; 2003. Disponible en http://ais.paho.org/sigepi/sp/ sigepi/download/Manual\%20de\% 20Usuario. pdf Acceso el 17 de agosto de 2010.

17. Organización Panamericana de la Salud. Clasificación estadística internacional de enfermedades y problemas relacionados con la salud $-10^{a}$ Revisión. Washington, D.C.: OPS; 1995 (Publicación Científica 554; vol 3).
Se identificó una situación de riesgo sanitario por exposición al arsénico asociado a la falta de control de calidad en la potabilidad del agua en la provincia de Buenos Aires.

El ICS constituyó un indicador efectivo para la caracterización del riesgo sanitario.

La exposición prolongada al arsénico puede derivar en la aparición de neoplasias malignas; sin embargo, la mortalidad por dichas patologías está asociada con el nivel de pobreza, que dificulta la aplicación de medidas preventivas para evitar la exposición y obstaculiza la atención médica oportuna en las etapas avanzadas de la enfermedad.

18. Organización Panamericana de la Salud. Área de Análisis de Salud y Sistemas de Información Sanitaria. EPIDAT. Programa para análisis epidemiológico de datos tabulado. Versión 3.0. Disponible en http://dxsp.sergas.es / ApliEdatos/Epidat/cas/default.asp Acceso el 17 de agosto de 2010.

19. Código Alimentario Argentino 2009. Bebidas hídricas, agua y agua gasificada. Disponible en http//www.anmat.gov.ar/códigoa/ capituloXII.pdf Acceso el 15 de julio de 2010.

20. Bocanegra OC, Bocanegra EM, Alvarez AA Arsénico en las aguas subterráneas: su impacto en la salud. Asociación Latinoamericana de Hidrología Subterránea para el Desarrollo (ALHSUD). VI ALHSUD-XXXII AIH Congreso Mar del Plata 2002. Disponible en http:/ / www.alhsud.com/public/articulos / Bocanegra2-Alvarez.pdf Acceso el 17 de agosto de 2010.

21. Blanes PS, Giménez MC, Herrera Ahuad CE Arsénico y otros elementos traza en aguas subterráneas en la región central de la provincia del Chaco. Universidad Nacional del Nordeste. Comunicaciones Científicas y Tecnológicas 2004. Disponible en http:/ / www. unne. edu.ar/Web/cyt/com2004/8-Exactas/E-070. pdf Acceso el 17 de agosto de 2010.

22. Penedo M, Zigaran A. Hidroarsenicismo en la provincia de Córdoba: actualización del mapa de riesgo e incidencia. Asociación Peruana de Ingeniería Sanitaria y Ambiental; AIDIS. Gestión ambiental en el siglo XXI. Lima, APIS, 1998, p. 1-20 Mapas, tab. Disponible en http://www.bvsde.paho.org/bvsaidis/ impactos/peru/argsam024.pdf Acceso el 17 de agosto de 2010.

23. Lerda D. Arsenic and cancer induction in Cordoba, Argentina. Aquatic arsenic toxicity and treatment. Leiden: Purphy and Guo Eds; 2003. Pp. 77-83.

24. Concha G, Nermell B, Vahter M. Metabolism of inorganic arsenic in children with chronic high arsenic exposure in northern Argentina. Environ Health Perspect. 1998;106: 355-9. 
25. Concha G, Nermell B, Vahter M. Spatial and Temporal Variations in Arsenic Exposure via Drinking-water in Northern Argentina. J Health Popul Nutr. 2006;24:317-26.

26. Guber RS, Tefaha L, Arias N, Sandoval N, Toledo R, Fernández M, Bellomio C, Martínez M, Soria de González A. Contenido de arsénico en el agua de consumo en Leales y Graneros (Provincia de Tucumán, Argentina). Acta Bioquímica Clínica Latinoamericana. 2009;43:201-7.

27. Vázquez H, Ortolani V, Rizzo G, Bachur J, Pidustwa V. Arsénico en aguas subterráneas. Criterios para la adopción de límites tolerables. XXVII Congresso Interamericano de Engenharia Sanitária e Ambiental, Porto Alegre, Brazil. 2000 Disponible en http:// www.bvsde.paho.org/bvsaidis/caliagua/ v-090.pdf Acceso el 17 de agosto de 2010.

28. Smedley PL, Kinniburgh DG. A review of the source, behaviour and distribution of arsenic in natural waters. Appl Geochem. 2002;17: 517-68.

29. Bhattacharya Bhattacharya P, Claesson M, Bundschuh J, Sracek O, Fagerberg K, Jacks G, Martin R, Storniolo A, Thir J. Distribution and mobility of arsenic in the Rio Dulce alluvial aquifers in Santiago del Estero Province, Argentina. Sci Total Environ. 2006;358: 97-120.

30. Matos, E., Loria, D.I., Vilensky M. Cancer Mortality and Poverty in Argentina: A Geographical Correlation Study. Cancer Epidemiology, Biomarkers \& Prevention. 1994;3: 213-8.

31. International Agency for Research on Cancer. Monographs Vol 84. Some Drinking-Water Disinfectants and Contaminants, including Arsenic related nitrosamines; 2004. Dispo- nible en http://monographs.iarc.fr/ENG/ Monographs/vol84/mono84-6A.pdf Acceso el 10 de junio de 2010.

32. Chiow HY, Chiou ST, Hsu YH, Chou YL, Tseng $\mathrm{CH}$, Wei ML, et al. Incidence of transitional cell carcinoma and arsenic in drinking water. A Follow-up study of 8,102 residentes in an arseniasis-endemic area in northeastern Taiwan. Am J Epidemiol. 2008;153:5411-8.

33. Tapio S, Grosche B. Arsenic in the aetiology of cancer. Mutat Res. 2006;612:215-46.

34. Mink PJ, Alexander DD, Barraj LM, Kelsh MA, Tsuji JS. Low-level arsenic exposure in drinking water and bladder cancer: A review and meta-analysis. Regul Toxicol Pharmacol. 2008;52(3):299-310.

35. Rivara M, Cebrian M, Corey G., Hernandez $\mathrm{M}$, Romiew I. Cancer risk in an arseniccontaminated area of Chile. Toxicol Ind Health. 1997;13:321-37.

36. Buchet JP, Lison D. Mortality by cancer in groups of the Belgian population with a moderately increased intake of arsenic. Int Arch Occup Environ Health. 1998;71:125-30.

37. Instituto Nacional de Estadística y Censo, Argentina. Prevalencia de vida, prevalencia de consumo actual, prevalencia de ex consumo y prevalencia de consumo pasivo de tabaco de la población de 18 años y más por sexo, grupo de edad, nivel de instrucción y provincia. Localidades de 5.000 y más habitantes. Total del país; 2005. Disponible en http://www.indec.mecon.ar/ Acceso en febrero de 2011.

38. Mosaferi M, Yunesian M, Dastgiri S, Mesdsaghinia A, Esmailnasab N. Prevalence of skin lesions and exposure to arsenic in drinking water in Iran. Sci Total Environ. 2007;390:69-76.
39. Chen CJ, Hsu LI, Wang CH, Shih WL, Hsu $\mathrm{YH}$, Tseng MP, et al. Biomarkers of exposure, effect, and susceptibility of arsenic-induced health hazards in Taiwan. Toxicol Appl Pharmacol. 2005;206(2):198-206.

40. Lindberg A, Ekstrom E, Nermell B, Rahman M, Lonnerdal B, Persson L, Vahter M. Gender and age differences in the metabolism of inorganic arsenic in a highly exposed population in Bangladesh. Environ Res. 2008;106: $110-20$.

41. Hopenhayn-Rich C, Biggs ML, Fuchs A, Bergoglio R, Tello EE, Nicolli H, et al. Bladder cancer mortality associated with arsenic in drinking water in Argentina. Epidemiology. 1996;7:117-24.

42. Smith AH, Goycolea M, Haque R, Biggs ML. Marked increase in bladder and lung cancer mortality in a region of Northern Chile due to arsenic in drinking water. Am J Epidemiol. 1998;147:660-9.

43. Ferreccio C, Gonzalez C, Milosavjlevic V, Marshall G, Sancha AM, Smith AH. Lung cancer and arsenic concentrations in drinking water in Chile. Epidemiology. 2000;11:673-9.

44. Marshall G, Ferreccio C, Yuan Y, Bates MN, Steinmaus C, Selvin S, et al. Fifty-year study of lung and bladder cancer mortality in Chile related to arsenic in drinking water. J Natl Cancer Inst. 2007;99:920-8.

Manuscrito recibido el 27 de octubre de 2010. Aceptado para publicación, tras revisión, el 14 de junio de 2011.
ABSTRACT

\section{Health risk for the vulnerable population exposed to arsenic in the province of Buenos Aires, Argentina}

Key words
Objective. To analyze the concentration of arsenic in water collected in localities of the province of Buenos Aires, Argentina, and the epidemiological relationship of that concentration to factors of susceptibility and associated pathologies.

Methods. In 152 samples from 52 localities of Buenos Aires from 2003-2008, the concentration of arsenic was quantified through the generation of hydride spectrophotometry of atomic absorption. A composite index of health $(\mathrm{CIH})$ was constructed using the content of arsenic and the percentages of households with unmet basic needs and dwellings without access to the potable water. Through the $\mathrm{CIH}$, risk areas associated with mortality from malignant neoplasms related to arsenic were defined. Results. Concentrations of arsenic spanned a broad range from 0.3 to $187 \mathrm{mg} / \mathrm{L}$, with a median of $40 \mathrm{mg} / \mathrm{L}$. Of the samples, $82 \%$ presented levels of arsenic higher than the acceptable limit of $10 \mathrm{mg} / \mathrm{L}$, and more than half of those came from households with potable water connections. In the departments studied, the average mortality (deaths/100 000 inhabitants) from tumors was greater in men than in women: respiratory tract (310 versus 76 ), urinary tract ( 44 versus 11 ), and skin ( 21 versus 11 ), respectively. The regions with greater concentrations of arsenic and of poverty, together with the lack of potable water connections, had a two-to-four times greater risk.

Conclusions. The findings from the composite index of health summarized the health risk from exposure to arsenic for lower socioeconomic levels of the population for a broad area of the province of Buenos Aires.

Arsenic; arsenic poisoning; risk assessment; water contamination control; poverty areas; Argentina. 